\title{
Math-Based Design of Sensing Bladders
}

Peter J. Schubert Packer Engineering, Inc.

Roopesh Saxena, Sachi Pinagapani and Madana Gopal

Delphi Electronics \& Safety 
The Engineering Meetings Board has approved this paper for publication. It has successfully completed SAE's peer review process under the supervision of the session organizer. This process requires a minimum of three (3) reviews by industry experts.

All rights reserved. No part of this publication may be reproduced, stored in a retrieval system, or transmitted, in any form or by any means, electronic, mechanical, photocopying, recording, or otherwise, without the prior written permission of SAE.

For permission and licensing requests contact:

SAE Permissions
400 Commonwealth Drive
Warrendale, PA 15096-0001-USA
Email: permissions@ @ sae.org
Tel: $\quad 724-772-4028$
Fax: $\quad 724-776-3036$

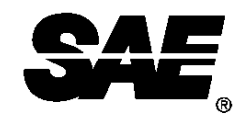

Global Mobility Database ${ }^{\circledast}$

All SAE papers, standards, and selected books are abstracted and indexed in the Global Mobility Database.

For multiple print copies contact:

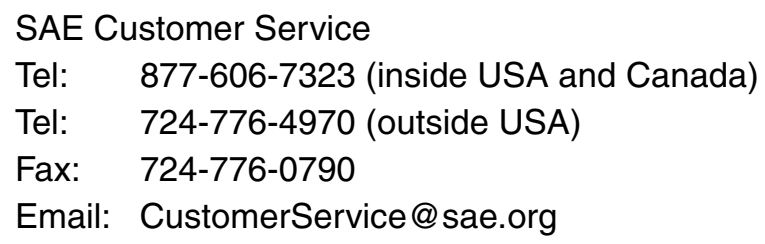

\section{ISSN 0148-7191}

\section{Copyright $\odot 2006$ SAE International}

Positions and opinions advanced in this paper are those of the author(s) and not necessarily those of SAE. The author is solely responsible for the content of the paper. A process is available by which discussions will be printed with the paper if it is published in SAE Transactions.

Persons wishing to submit papers to be considered for presentation or publication by SAE should send the manuscript or a 300 word abstract to Secretary, Engineering Meetings Board, SAE.

\section{Printed in USA}


Math-Based Design of Sensing Bladders

Peter J. Schubert

Packer Engineering, Inc.

\author{
Roopesh Saxena, Sachi Pinagapani and Madana Gopal \\ Delphi Electronics \& Safety
}

Copyright @ 2006 SAE International

Keywords: Passive Occupant Detection System, pressurebased occupant sensing, airbag suppression.

\begin{abstract}
Through the use of finite-element modeling, pressure patterns on the underside of seat foam can be computed for a variety of occupants and seating positions. A design tool has been created which allows an engineer to evaluate different layouts for a pressure-sensing bladder in just minutes. This is important to meet FMVSS-208 safety regulations for vehicles sold in the US. Further, an artificial intelligence search engine has been applied to this problem to achieve near-optimal performance given the constraints of the seat design. Results are shown and compared with the traditional manual method of layout design.
\end{abstract}

\section{PROBLEM DESCRIPTION}

One of the key provisions to meet the National Highway Traffic and Safety Administration's (NHTSA) frontal FMVSS 208 (Federal Motor Vehicle Safety Standard) safety criteria is Option-1 known as "suppression" [1]. The intent here is to suppress the frontal passenger airbag(s) in the presence of an infant or a child to 'minimize the risk of injuries and deaths caused by airbags.' In general, this size-based sensing system provision allows the manufacturers to demonstrate suppression in a static condition for various infant/child seats and seated child configurations [2,3].

Among the many solution options to help meet the regulation, Delphi's Passive Occupant Detection System for seat's B-surface (PODS-B, located underneath the seat foam), addresses this challenge with a fluid-filled bladder and an associated sensing and command system. The product works by detecting pressure changes in the presence of occupants, non-occupants, child seats, seated children and adults in multiple seated configurations.

This paper discusses the design of PODS-B with mathbased tools LS-DYNA 3D (Finite Element Analysis) and FETool2K (in-house bladder designer tool). The first software is used to calculate the pressure on the bladder in response to the applied load on the 'A-surface'. The latter tool, a PC-based software specifically developed in-house, converts the FE results to voltage changes (based on interface pressure data) to predict the output of the system. Figure 1 shows an exploded view of the FEA components.

A special challenge for the sensor design is separating the 6 -year old seated child from a $5^{\text {th }}$ percentile adult female occupant. The goal of a good bladder design is to maximize the ability of the sensing system to discriminate between them. To address this, butt forms representing these two classes of occupants have been developed to generate the 'A-surface' loading condition for multiple seated conditions. For the analysis, there are two sets of loading used in the design for the pressure calculations. The first includes the 'FMVSS 208 ' positions as specified in the final rule. The second set includes internally-generated targets.

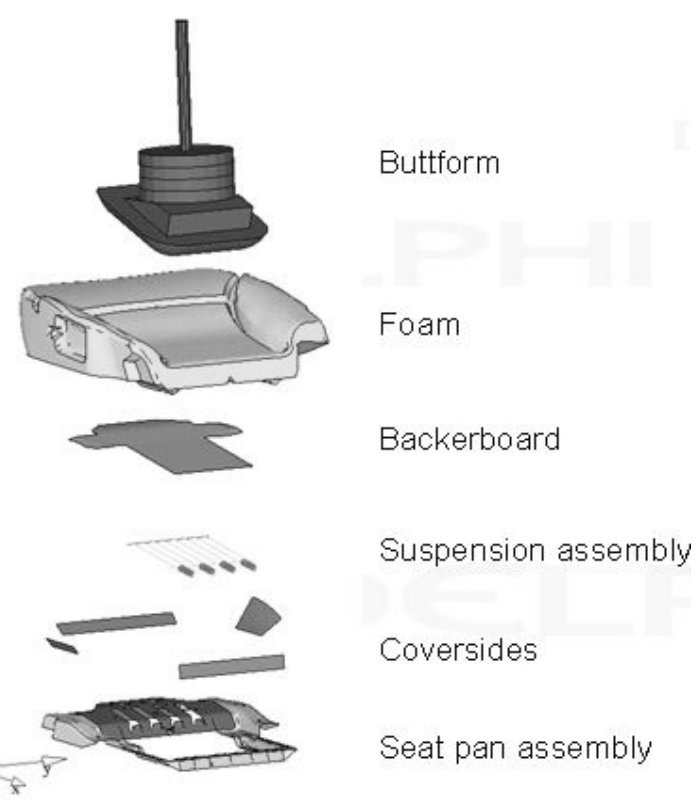

Figure 1. Exploded view of seat bottom components and occupant butt-form. 
Designing a bladder to maximize separation between inhibit and enable cases involves many constraints and tradeoffs. Studies of occupant in vehicle seats [4] show that occupant seated weight can differ from standing weight through off-loading onto the vehicle floor, door arm-rests or the front dash board. While normallyseated occupants dominate, there is a nearly-infinite variety of seating postures possible, making it very difficult to accurately sense and classify the occupant in every case. Objects pushed beneath the seat may also apply upward pressure on the suspension, leading to erroneous readings. Seat packaging is a complex task which includes multiple subsystems for seated comfort, seat positioning, seat HVAC, and crashworthiness. These systems and the connections among them place restrictions on mechanical interferences, thickness, hardness, and location of pressure-detecting sensors. Bladder design, subject to these constraints, aims to cover a wide range of seating postures for the full range of human body sizes and types, and provide clear separation of 6 year old children from $5^{\text {th }}$ percentile females, the bracketing cases for inhibit and deploy classifications.

\section{METHODOLOGY}

The original method for bladder design involved considerable trial and error. With greater experience, certain design practices became accepted. Detailed guidelines were written and used to assess new designs. A further advancement is the use of pressure-sensitive mats placed at the seat B-surface under a battery of occupant test cases to determine pressure patterns. Using these pressure patterns, bladder design engineers can locate regions of the B-surface more activated by a child compared to an adult (e.g. the center and front edge) and vice versa (e.g. edges of the seat and at the anti-submarining structure). By adjusting bladder sensitivity in the various regions, the number of design iterations has been reduced. The goal of this work is to advance a step further, to generate a virtual model of the seat so that bladders can be designed in a single pass, in advance of prototype seat delivery. By using mathbased methods, we have been able to demonstrate a faster design cycle with improved ability to properly classify seated occupants.

\section{SEATS}

The design methodology for PODS-B begins with finite element analysis (FEA) of the seat bottom using LSDYNA. The program Hypermesh is used as preprocessor to define grid points, and LS-Post is used as a post-processor for animation and analysis.

Within the FEA simulation, the butt-form of $5^{\text {th }}$ percentile and 6 year old occupant are dropped onto the trimcovered foam from a height of $25 \mathrm{~mm}$ with $1 \mathrm{~g}$ of acceleration applied. The system is run 150 milliseconds (ms), at which time the pressure pattern is captured from a thin, flexible plate placed between the foam bun and the seat suspension. Seat components which are not in direct contact with the foam such as frame parts below the seat pan/suspension are not modeled as their contribution to B-surface sensing can be considered insignificant. When we place the butt-form on the seat for any of its positions the butt-form does not interact with the seat back, hence the seat back is modeled only for those situations where the child seat engages the head rest (e.g. Century Next Step Booster Seat).

The parts that are modeled include the seat pan, seat pan reinforcements, spring suspension, seat foam, seat cover (trim) and butt-form. Spring suspension is not modeled in pan-type seats where springs and wires are not present under the foam bun.

The seat pan material (usually steel) is considered rigid as they are very stiff compared to the foam. The appropriate spring steel properties are defined for the spring and wire suspension bed (if any). Foam material properties are input as a stress-strain curve, such as the two examples shown in Figure 2. The butt-form is defined as a rigid material.

Foam Stress Vs Strain

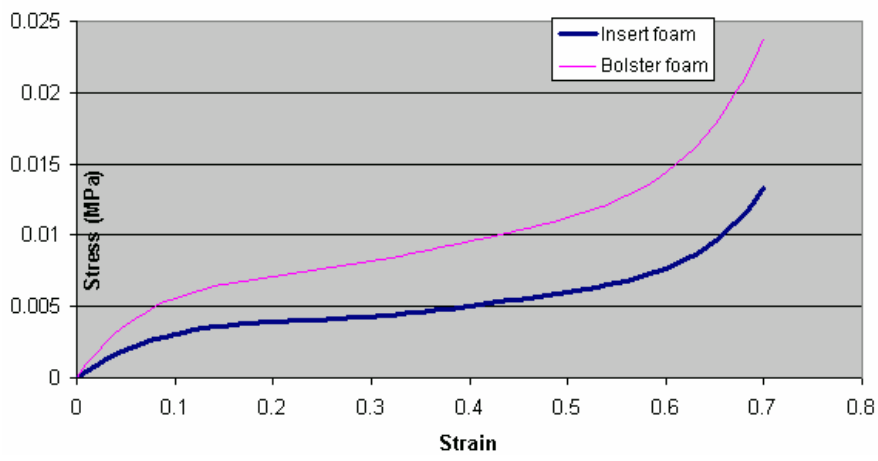

Figure 2. Stress-strain curves for two foam hardnesses.

The design flow using math-based tool takes approximately 6 weeks, and shown in Figure 3 . The process begins with the receipt of computer-aided design (CAD) data. CAD data direct from the design tool is preferred, however, a digitizing scan may be used in those cases where a completed seat is already available. Meshing the foam bun is critical for good results. Interfaces between materials deserves special attention since the significant amount of deflection in the foam bun can lead to node instabilities unless care is taken during meshing.

Simulation runs involve three phases. First, suspension springs are pre-tensioned. Springs have an initial tension before the occupant touches the seat, which is modeled by pulling the spring to its nominal length at the start of the simulation. Next, the seat trim cover is pulled to its installed length. In the physical seat, the cover is affixed using J-clips (or other mechanism) onto the seat frame, putting tension into the trim material. To replicate this effect, pre-simulation is performed on the seat cover by pulling the cover tabs similar to the physical installation. In the final phase, the occupant model is dropped into the desired location. Modeling the drop is 
an important connection to real-world testing since the reverberations tend to unlock static friction between surfaces, and give a reliable and repeatable rest state. Figure 4 shows a semi-transparent cut-away view (side and rear) of the $5^{\text {th }}$ percentile female B-form being dropped in the normally-seated position into a seat bottom assembly.

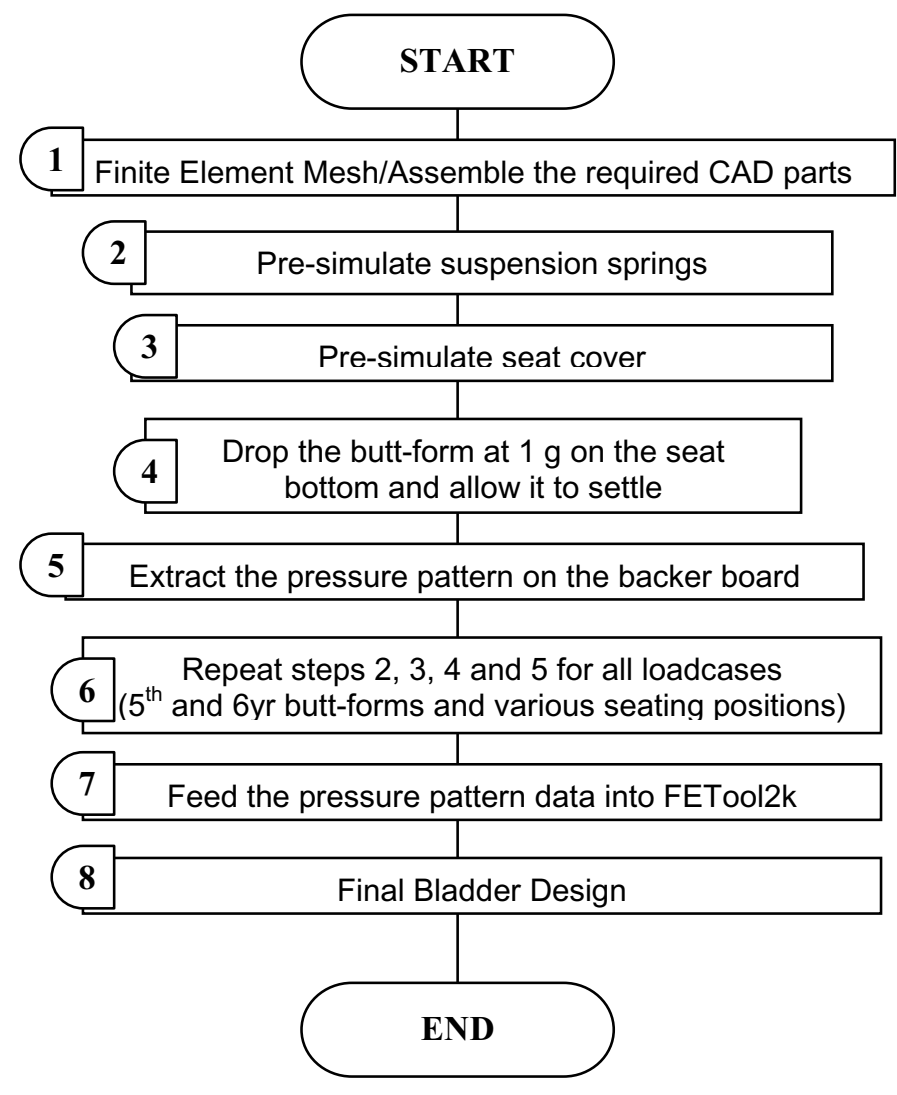

Figure 3. Design process flow for one-pass bladder design.

This process is repeated for each seating scenario considered for analysis. Batch operation allows an entire series of scenarios to be run unaided to better utilize computer time and license availability. Typically the model includes 25,000 nodal points and requires 8 hours of computer time for each scenario.

Each seating scenario results in a force pattern applied to a "backer-board" inserted between the foam and the suspension or pan. The backer-board is used for puncture resistance, and as a reaction surface, for the silicone-filled bladder in production systems. Force patterns on this surface are considered representative of what the bladder will experience. The silicone-filled bladder itself is not modeled. This omission was based on the complexity of the fluid-structure interaction problem that would be required for first-principles modeling, and because the compliance of the bladder is expected to have minimal effect on the B-surface pressure pattern.

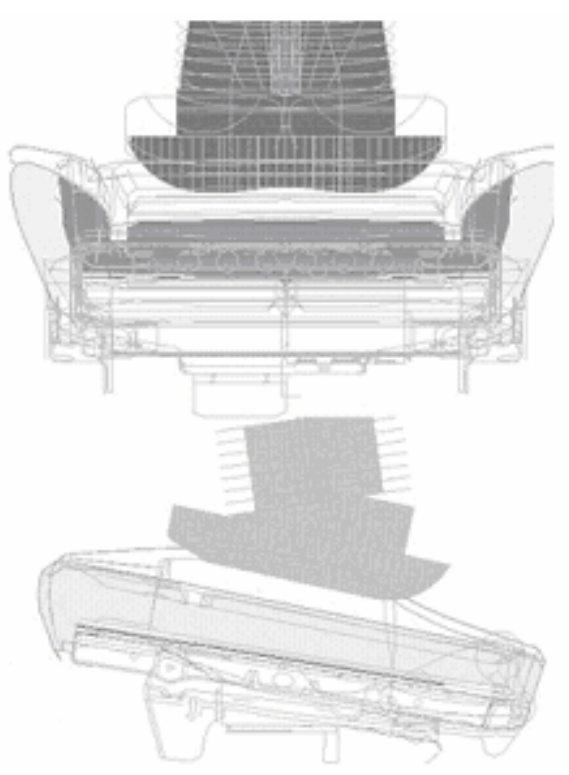

Figure 4. Semi-transparent cross sections showing buttform suspended over trim-covered foam at the start of the FEA run.

\section{OCCUPANTS}

When an occupant enters a vehicle seat, there is a pressure imposed on the backer-board whose magnitude and distribution is a function of the occupant size and seating posture. The shape of the pattern is complex, and depends upon many factors. In keeping with a reasonable problem definition, a few simplifying assumptions have been made. First, only the pelvis and upper legs of the occupant are used, since the rest of the body does not directly interact with the seat cushion. Second, biofidelic occupant models are still maturing, so we have approximated the appropriate portions of human anatomy with rigid butt-forms. This practice also reduces computation time. The butt-form for the $5^{\text {th }}$ percentile female was derived from a test subject who closely-matched the standard definitions for that category [6]. The 6 year old butt-form was adapted from the $5^{\text {th }}$ percentile female butt-form by narrowing the hips to the standard governmental guidelines for a 6 year old, and by extending the femurs to match the length of a 6 year old anthropomorphic test device (ATD). Perspective views of these two butt-forms are shown in figure 5 .

The effective pressure applied by a butt-form on seat cushion does not equate to the total occupant weight because of weight loss attributable to leaning on the seatback and feet resting on floor. Seated weights, determined by internal testing, are used to load the buttforms. A seated weight of $80 \mathrm{lbs}$ is used for the smallstature female, and a seated weight of $50 \mathrm{lbs}$ is used for the child. 


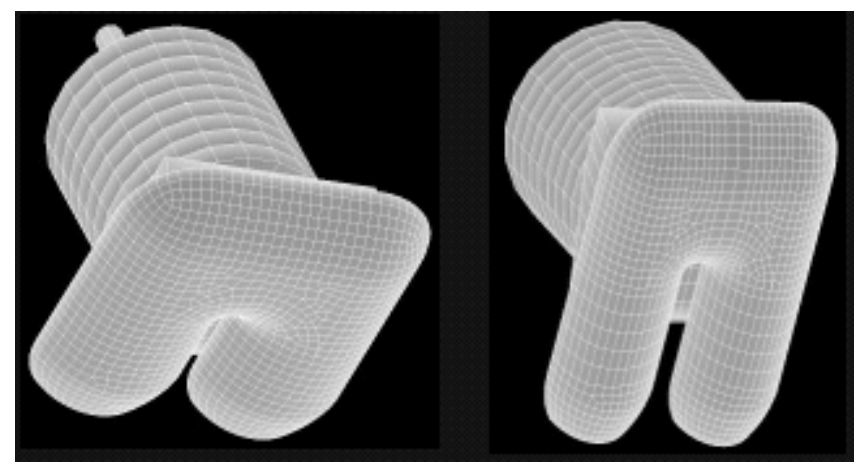

Figure 5. Perspective view of butt-forms used for simulation: $5^{\text {th }}$ percentile female (left), and 6 year old child with full-length thighs (right). The stacked cylinders represent brass weights used to match prototypical occupant weight.

The butt-form is required to load the seat cushion at $1 \mathrm{~g}$ to simulate a seated occupant. The backer-board force pattern is measured when butt-form loading reaches an equilibrium state. However, simply allowing the buttform to settle under gravity alone requires long computation times. To reach the equilibrium state more quickly, the butt-form is applied as a prescribed load in a short time as shown in Figure 6. Total analysis time consists of $30 \mathrm{~ms}$ of pre-simulation, 60 milliseconds (ms) for prescribed load on butt-form, and another $60 \mathrm{~ms}$ to achieve equilibrium state. Pressure pattern results are output at the end of the $150 \mathrm{~ms}$ simulation time.

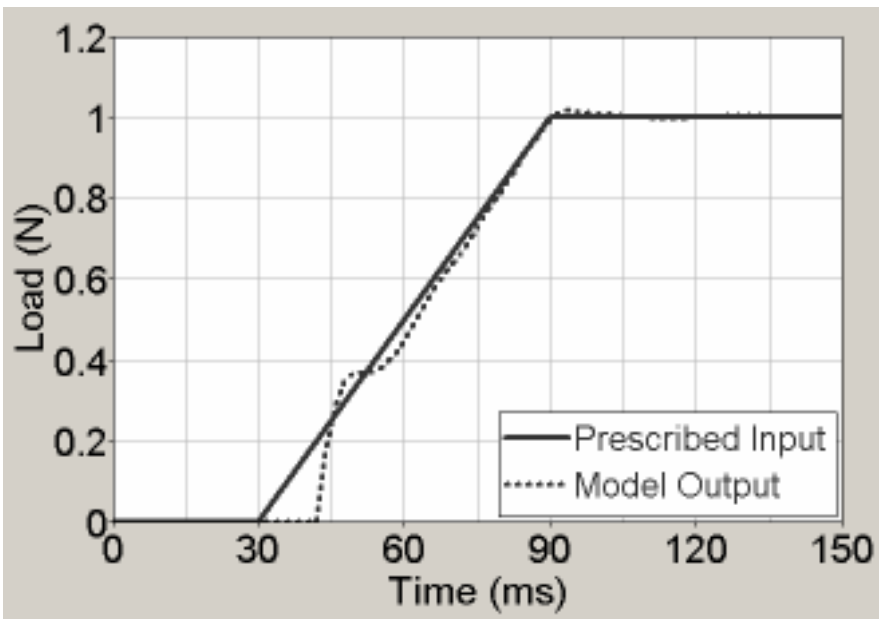

Figure 6. Prescribed loading schedule to minimize overshoot and reduce computation times. The dashed plot overlay shows the model output.

Determining the occupant scenarios to use is vitally important to successful bladder design and system performance. Balancing the need for many test cases with the desire to work efficiently, we have settled on an approximate number of positions between 13 and 16 . This data was drawn from three sources. First, the top five conditions were taken from a study [4] of occupant seating postures for adults. Second, the key FMVSS 208 positions were included. Finally, certain seating scenarios have been found to be especially difficult through many years of field testing, and these were included as well.

The use of butt-forms is clearly a crude approximation to a real human, although they are computationally attractive. A number of techniques were used to improve fidelity of the final results, such as angling of the weight stack to represent torso weight correctly applied to the pelvis. The trim material of the seat cover can partly suspend the occupant, and this effect has been included. On-going work to further extend biofidelity is covered in the final section of this paper.

\section{BLADDER DESIGN}

The collected B-surface pressure patterns from the last time of the FEA run are gathered digitally and spatially filtered to a larger mesh size. The minimum design element in a bladder is 1 inch, so we interpolate the 9.4 $\mathrm{mm}$ FEA grid (selected to reduce aliasing effects) to a $25.4 \mathrm{~mm}$ bladder design grid. All pressure patterns from the enable conditions (adult) are grouped together and averaged. The identical averaging process is also applied to the inhibit group (children and other cases where airbag deployments are unwanted). By subtracting the second group average from the first, a point-by-point force difference surface is created at the backer-board location. Figure 7 shows one such plot where positive values arise from enable and negative values come from inhibit scenarios.

The bladder design process involves judicious placement of active bladder cells, subject to the constraint that they all be connected, and all connect with the common port in the center of the bladder. There is no unique best answer, so a variety of designs are evaluated.

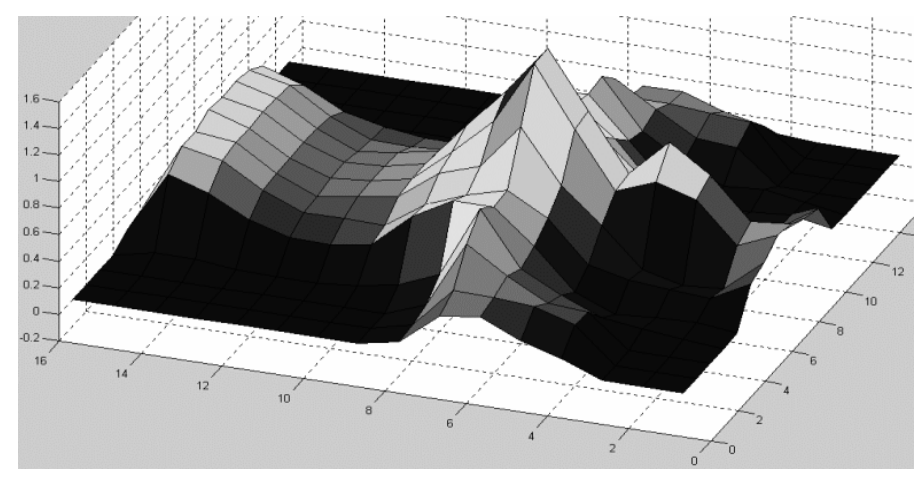

Figure 7. Difference surface between averaged force patterns for inhibit and enable conditions.

Using a graphical tool (FETool2k), the designer creates a bladder layout based on the pressure difference surface. The most significant time and cost savings of this method is that bladder designs can be evaluated in seconds, instead of weeks or months. With a model of the bladder response to the pressure patterns, subject to 
the designer's layout, we can estimate the separation metric between occupant classifications.

A simple model of the pressure transducer converts the bladder signal to a voltage. A further model of the analog to digital conversion process gives a microcontroller-friendly input in integer counts on a range between 0 and 255 (8-bit CPU). By running each seating scenario through the bladder design (FETool2k), and then applying the bladder model, the sensing system "counts" can be predicted. The most basic performance metric is the separation (in counts) between the $5^{\text {th }}$ percentile female and the 6 year old child in a normally seated position. In general, the accepted practice at Delphi is to group all enables and all inhibit scenarios, find the minimum of the former and the maximum of the latter, then define the separation as the difference between them.

Using FETool2k, two or three bladder designs will often emerge as nearly-equivalent. Provided these top designs are not trivially different, each design will be built and tested. Results are shown in the next section.

\section{RESULTS}

As of this writing, bladder designs for four vehicle platforms have been successfully completed using this math-based design methodology. For one such platform, the normally-seated separation between $5^{\text {th }}$ female and 6 year old are shown for four design options. The first one, "MANUAL" is the test result using the production-intent bladder, created using the conventional manual method. The "FULL SIZE" result is for a bladder of the maximum size allowed by the geometry and interferences of the seat, and is a design which is often used as a starting point for new platforms. Designs labeled "A" and "B" in Figure 9 were derived exclusively using the methods described above. The two sets of vertical bars for each design are the simulated results (labeled "SIM", with grid fill) and the laboratory test results ("TEST" with grey fill).

Two important observations may be derived from Figure 9. First, the trend between test and simulation is fairly good, even though the absolute agreement is not precise. Second, the design "A" out-performed the manual design in both simulation and test. Since the goal of this methodology is to design a better bladder, this result is key. Another observation is that design " $\mathrm{B}$ " was modestly better than the manual design in test, but the simulation showed it would perform slightly worse. We have learned that lab test results tend to have a nonnegligible error, and that bladders may test better or worse depending on the details of how they are installed in the seat. Clearly, validation of any design is critical.

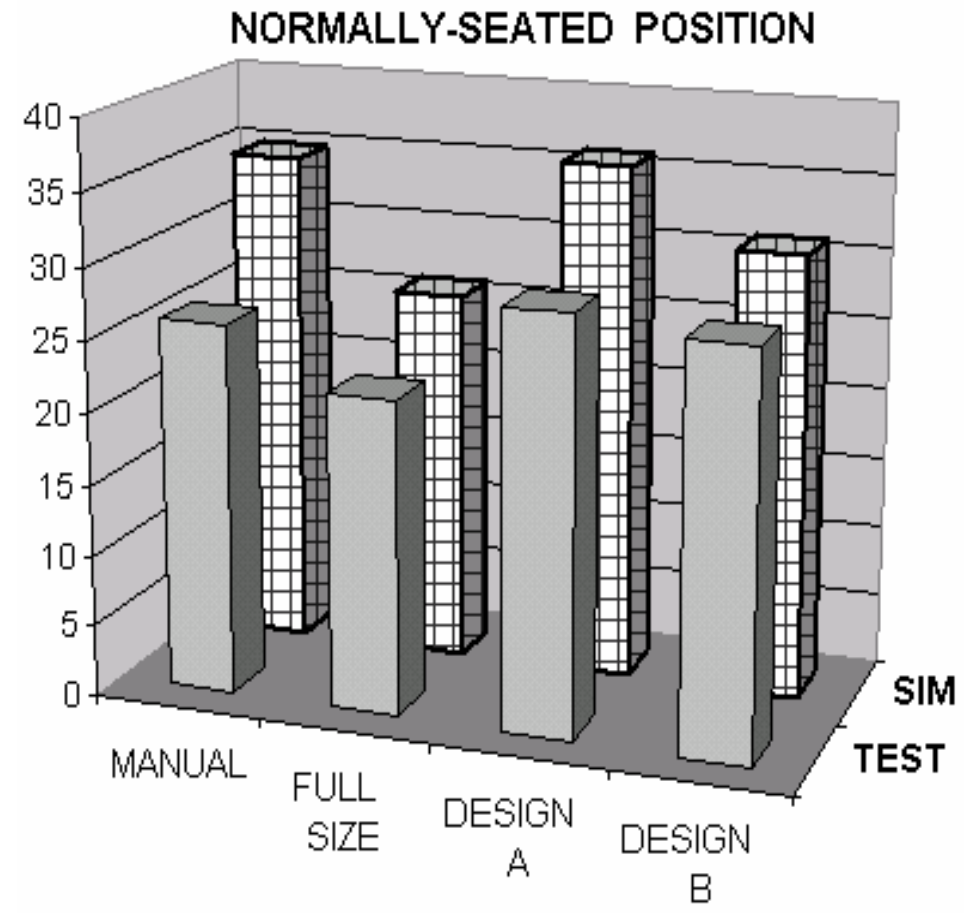

Figure 9. Comparison of simulation and test results for separation counts between the $5^{\text {th }}$ percentile female and the 6 year old child in a normally-seated position. A count is approximately equal to $1 \mathrm{lb}$.

Results comparing the full set of occupant seating scenarios across four vehicle platforms is shown in Table 1. The separation numbers, cited in integer counts, are approximately equal to $1 \mathrm{lb}$, so that a value of 10 would indicate $10 \mathrm{lbs}$ of sensed difference between the lightest enable case (small-stature female) and the heaviest inhibit case (child, or occupied and cinched child seat). For this cross section of vehicle types, it can be seen that the new methodology described here improves from -1 to +6 compared to the productionintent manual designs, with an average gain of 2.5 counts. An interesting observation is that the standard deviation of the new design method (math-based) for the Sedan case is three times smaller than the manual design. Therefore, from a signal-to-noise perspective, designs using the new method may be even more favorable.

Another factor to consider is test measurement variation. Best-case repeatability is considered to be $+/-1$ count of variation. Therefore, differences smaller than this amount should be considered within experimental tolerance. Overall, it can be seen that the new methodology is at worst on par with the manual design process, or at least offers several counts of improvement. 


\begin{tabular}{|l|c|c|c|}
\hline PLATFORM & MANUAL & MATH & IMPROVEMENT \\
\hline Truck - Full & 10 & 10 & 0 \\
\hline Van & 27 & 32 & 5 \\
\hline Sedan & 41 & 40 & -1 \\
\hline Truck - Sm & 6 & 12 & 6 \\
\hline
\end{tabular}

either $5^{\text {th }}$ percentile females or 6 year old children. The counts are shown on the ordinate axis, and the occupant serial number is shown on the abscissa. Separation, defined in the worst-case sense described above is 20 counts. The one point circled is a statistical outlier (verified using MINITAB). With this removed, the separation is 28 counts, which compares reasonably well with the math-based prediction of 32 counts, and which out-performed the manual design.

\section{Live Clinic Testing}

Table 1. Separation results (in counts) of 4 seat platforms comparing conventionally-designed bladders (MANUAL) with bladders designed using the math-based methodology (MATH) for 12 occupant seating scenarios.

\section{VALIDATION}

Validation of computer models with known test cases is important to justify the assumptions made. Obtaining reliable data to support the force patterns at the foam Bsurface turned out to be a daunting challenge. One method is to use a force-sensitive array, such as the Vista Medical FSA mat. Unfortunately, force-sensing mats, when installed into a vehicle seat, experience pinch points, bunching, and topographically-induced force concentration. Figure 10 shows side-by-side comparison of B-surface force patterns from a sensor array and using FEA. On the left plot, numerous spikes are seen, and adjacent points can be found varying by two orders of magnitude. Various techniques were attempted to improve the sensor array data, such as spatial filtering and outlier removal, without success. Even a repeatability study to re-install the mat between readings, and then taking bounded averages point by point failed to give a coherent pressure pattern. We were left unable to adequately verify B-surface pressure patterns.
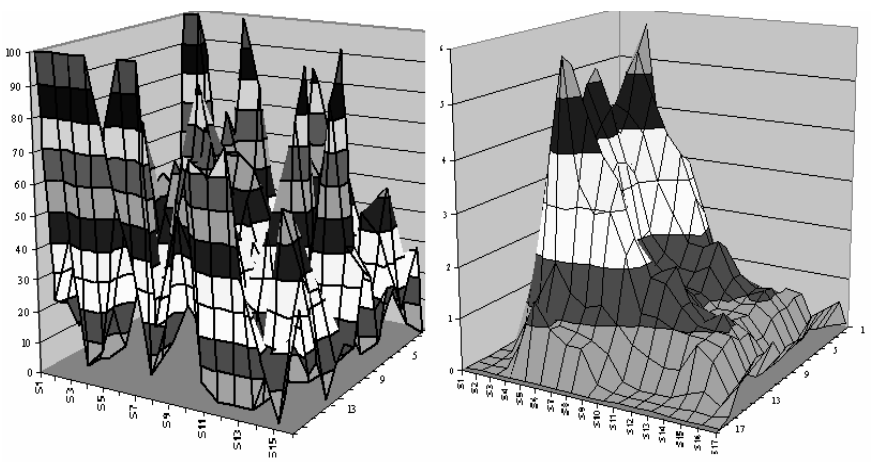

Figure 10. Comparison of force mat (left) and FEA (right) pressure patterns at the foam B-surface.

Without intermediate validation, we turned instead to a final validation using live clinic results. This is one of the practices recommended in the FMVSS 208 standard, and is done routinely in Delphi labs. Figure 11 shows validation results using live occupants categorized as

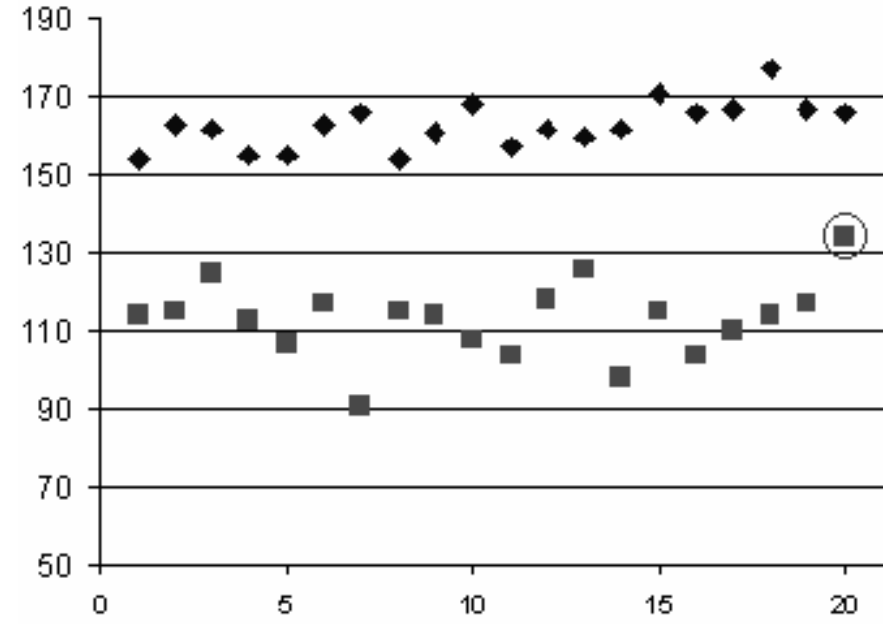

Figure 11. Live clinic results showing $5^{\text {th }}$ percentile females (diamonds) and 6 year old children (squares). Separation is measured as the minimum number of counts between classes. The circled point is a statistical outlier.

\section{OPTIMIZATION}

An important question to be asked when designing an occupant classification bladder is: How do you know when you have achieved the optimum performance? As stated above, there is no single correct answer.

However, there are methods available to estimate the maximum performance capability given the seat design, the force patterns from selected scenarios, and the constraints applied to bladder design. To arrive at an answer to this question, we applied a genetic algorithm (GA) search tool $[5,7]$ to the bladder design process.

Figure 12 shows a computer-generated optimal results for two different vehicle platforms, based on the prediction of separation for 12 occupant/seating scenarios, subject to the constraint that all fluid-filled regions must have connectivity to the center 4 cells where the pressure sensor port is located (cells H8-19 in the rightmost figure). To minimize computation time, the GA was seeded with designs developed using the FETool2k utility described above, and allowed to optimize further. 

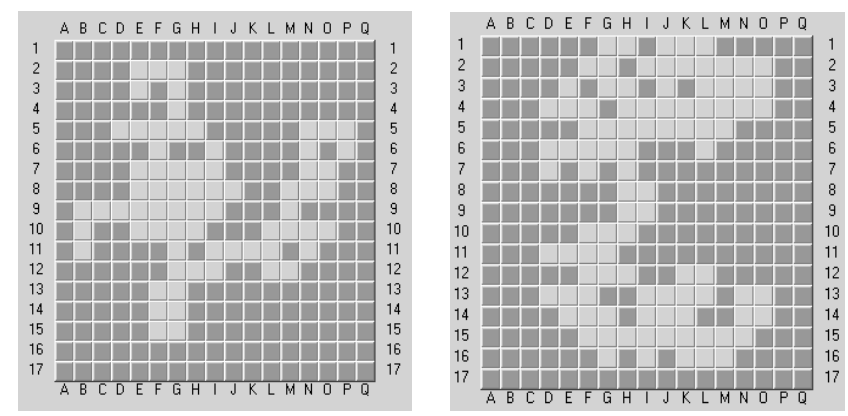

Figure 12. Two computer-generated designs for the same platform, subject to a connectivity constraint.

This design technique produced designs with predicted separations several counts higher than the FETool2kgenerated designs. However, upon testing in both cases, the actual results were within 1 count of the separation from the FETool2k-generated designs.

\section{DISCUSSION}

Acquiring the CAD data needed to begin this process can be challenging. First, seat designs change often, and are frequently being re-designed so no accurate CAD data is available; and secondly not all changes are communicated in a timely fashion. This step tends to get more complicated once you have a vehicle manufacturer and multiple suppliers involved in the design process.

Implementing a semi-automated design process across a world-wide production environment is a daunting challenge. Regional sites often prefer home-grown solutions tailored to their work style and customer inputs. Automation is not always accepted or in general considered a first choice methodology by design engineers. Ramp-up of the skill set needed for FEA work is another consideration. These issues have been addressed with lessons learned and thorough documentation, plus making the tools as user-friendly as possible.

Achieving near-optimal bladder designs in a single pass is attractive to program managers on two fronts. First, the uncertainty in program schedules and resource allocation is greatly diminished. Second, the outcome from the new methodology is able to estimate maximum system performance and evaluate designs against that standard. In the case where a given seat design is not conducive to occupant classification, providing scientific evidence that the performance requirements are unachievable regardless of design is of considerable benefit in negotiating changes.

Estimating maximum performance through a GAoptimized design is useful for evaluating productionintent designs. However, at this stage of development, an artificial intelligence design routine does not always produce bladders which would be acceptable for manufacturing. As Figure 12 shows, the irregular and asymmetric designs suggest over-fitting to the set of seating scenarios used. This drawback can be overcome with more seating scenarios or more constraints on the GA search. However, the close agreement of FETool2k-generated designs with these GA-generated designs gives us reasonable assurance that the tools created for this work allow a designer to design a near-optimal bladder.

\section{NEXT STEPS}

Certain simplifying assumptions have been made to this approach. It will be appreciated by anyone familiar with modeling real-world systems from first principles that any model is an approximation to reality. At this state of development, a considerable body of experience has been developed which makes this method reasonably successful. And while no system can completely match real-world testing and predict every measurement subject to random testing error, it is a strong objective of this work to advance the fidelity of the models and more closely match test results.

The next two important steps for this work are to include CAD models of child seats and to modify the bladder model to account for partially-activated cells now favored by certain bladder designers. Child seat CAD models may be obtained from non-contact surface profiling, or directly from design files by the child seat manufacturers. Surface profiling is difficult because of topography and the need to turn a point cloud into a solid which can be meshed. Nonetheless, we have been able to model the bottom surface of known-difficult child seats and are working now to incorporate these into our modeling runs.

Biofidelic human models are available to a certain degree of accuracy. Math models of the Hybrid III ATD do not accurately capture human motion under the $1 \mathrm{~g}$ conditions used for occupant work. Human body models continue to improve, but were not used here because of the added complexity, which drives up computation times. On-going development work progresses step-wise towards a system which provides continuous improvement in a design methodology for an important automotive product.

\section{CONCLUSIONS}

We have presented a complete design methodology for pressure-sensing bladders based on mathematical modeling and computer-aided engineering tools. Our results have repeatedly demonstrated performance at least on par with, or on average better than, manual design methods. Our approach can be completed in 8 weeks, including bladder fabrication, compared to design times up to 6 months for the conventional approach. Achieving as-good or better performance in as-good or faster time with the ability to estimate design quality compared to an optimal value is a considerable accomplishment. Work is on-going to further deploy this method across a wider range of applications. 


\section{ACKNOWLEDGEMENTS}

Nirmal Narayanasamy contributed heavily to the early development work of the FEA models. Brad Stroup completed the bladder model as part of his Senior Thesis at Kettering University. Patrick Herber assisted with extension of the bladder model to account for gain changes with fill volume. Dr. Pankaj Mithal performed bladder model validation and is actively involved in next step research. The authors also gratefully acknowledge Kevin Kincaid for his sponsorhip of this work and useful guidance at key milestones.

\section{REFERENCES}

1. Final Rule for Advanced Airbags - DOT, NHTSA 49CFR Parts 552, 571, 585, and 595, [Docket No. NHTSA 00-7013, Notice 1] RIN 2127-AG70 'Federal Motor Vehicle Safety Standards; Occupant Crash Protection.' Section VIII, A \& B. Page 6. (www.nhtsa.gov).

2. Occupant Classification System for Automotive Airbag Suppression by Michael E. Farmer, Eaton Corporation, and Anil K. Jain, MSU. http://www.cse.msu.edu/prip/Files/FarmerJainCVPR 03.pdf

3. Development of Occupant Classification System for Advanced Airbag Requirements by Shigeyuki Nozumi, Mitsubishi Motors. http://www.mitsubishimotors.com/corporate/about us/technology/review/e /pdf/2004/16E 12.pdf

4. Zhang, L, Chen, L.; Vertiz, A.; Balci, R., "Survey of Front Passenger Posture Usage in Passenger Vehicles," SAE World Congress 2004, SAE 200401-0854.

5. Schubert, P, Loughlin, D., "Efficient Optimization of Large k Real-time Control Algorithm," Seventh International Workshop on Artificial Intelligence and Statistics, Ft. Lauderdale, FL, 1999.

6. NHTSA regulatory sizes for test subjects: http://www.nhtsa.dot.gov/cars/rules/import/FMVSS/\# P572

7. Goldberg, D.E., "Genetic Algorithms in Search, Optimization and Machine Learning:, Addison Wesley, 1989.

\section{CONTACT}

Peter J. Schubert, Ph.D.

Packer Engineering, Inc. 1950 N. Washington St.

Naperville, IL 60566-0353

630-577-1928 or 800-323-0114 